\title{
Partitions in the prime number maze
}

\author{
by \\ Michael I. Hartley (Klang)
}

1. Introduction. Paulsen ([6]) defined the "prime number maze" $G_{1}$ as follows. $G_{1}$ is a directed graph, whose vertices are the prime numbers. There is an edge in $G_{1}$ from $p$ to $q$ if and only if their binary representations have Hamming distance 1 , and if $3 p \geq q$. That is, if and only if the binary representations of $p$ and $q$ differ by exactly one digit, and that digit may only be the first digit of $q$ if we are merely prepending a single digit (1) to $p$. For example, there would be bidirectional edges between $61=111101_{2}$ and $53=110101_{2}$, and between $13=1101_{2}$ and $29=11101_{2}$. However, although there would be an edge from $37=100101_{2}$ to $5=101_{2}$, there is none from 5 back to 37. Nonetheless, there is a path from 5 to 37 , as pointed out in [6], as follows: $5 \rightarrow 13 \rightarrow 29 \rightarrow 61 \rightarrow 53 \rightarrow 37$.

The structure of the prime number maze appears to be quite intricate. Paulsen discovered ([6]) that the shortest path in this maze from 2 to 353 leads through primes exceeding 132 digits, then suddenly dropping through $2^{441}+2^{392}+2^{27}+353 \rightarrow 2^{392}+2^{27}+353 \rightarrow 2^{27}+353 \rightarrow 353$. In fact, there is a tiny chance that the shortest path to this number must go even higher, since Paulsen used Miller-Rabin strong pseudoprimality tests, rather than strict primality tests, on the larger numbers in the sequence.

Furthermore, Paulsen noted that $G_{1}^{\prime}=G_{1}-\{3\}$ is divided into at least two "partitions". Let $G_{p}$ be the subgraph of $G_{1}$ induced by the subset of all the vertices $q$ of $G_{1}$ for which there is a path from $p$ to $q$. Similarly define $G_{p}^{\prime}$. It turns out that if $11 \in G_{p}^{\prime}$, then $G_{p}^{\prime}$ and $G_{2}^{\prime}$ are disjoint, as may be proven using a simple argument involving what Paulsen calls the "parity" of a prime number.

Let $p>3$ be prime. We say $p$ has correct parity if either $p \equiv 2 \bmod 3$ and $p$ has an even number of 1 bits in its binary representation, or $p \equiv 1 \bmod 3$ and $p$ has an odd number of 1 bits in its binary representation. Otherwise, it has incorrect parity. Paulsen proved (Proposition 1 of [6]) that any edge in $G_{1}$ between $p, q>3$ must preserve parity. He uses this fact to show that

2000 Mathematics Subject Classification: 11A41, 11B05, 11R18. 
$G_{1}^{\prime}$ consists of at least two partitions, the $\alpha$-partition, containing 5 , and the $\beta$-partition, containing 11. Note that $G_{2}$ contains the $\alpha$-partition. All the primes in the $\alpha$-partition have correct parity, all those in the $\beta$-partition have incorrect parity.

There are other partitions as well, in fact from Proposition 3 of [6] we know that $G_{1}$ has infinitely many isolated points. The $\alpha$ - and $\beta$-partitions are of interest because they appear to be infinite.

In fact, Paulsen conjectured that there are more infinite partitions than just the $\alpha$ - and $\beta$-partitions. He noted the apparent existence of what he called the $\gamma$ - and $\delta$-partitions of $G_{1}^{\prime}$. Their main features are characterized in Table 1, which is also found in [6].

Table 1. The partitions as conjectured by Paulsen

\begin{tabular}{|c|c|c|c|}
\hline & $\begin{array}{c}\text { Lowest } \\
\text { prime }\end{array}$ & $\begin{array}{c}\text { Starting } \\
\text { point }\end{array}$ & Comments \\
\hline$\alpha$-partition & 2 & 2 & "main maze" \\
$\beta$-partition & 11 & 547 & can reach $\alpha$ via 3 \\
$\gamma$-partition & 277 & 4957 & incorrect parity \\
$\delta$-partition & 683 & 35759 & correct parity \\
\hline
\end{tabular}

Paulsen left it as an unsolved problem whether or not these are the only infinite partitions, and whether or not the $\gamma$ - and $\beta$-, or the $\alpha$ - and $\delta$-partitions eventually join up. Evidently, from parity arguments, the $\gamma$ partition cannot join up with $\alpha$ or $\delta$, nor $\delta$ with $\beta$, except possibly through the number 3 .

Later in this article, these unanswered questions will be settled, and we will see a more satisfactory way to delineate the partitions.

2. The base $b$ mazes. In order that the results may be stronger, we first of all define the base $b$ maze $G_{*}^{b}$ as follows.

$G=G_{*}^{b}$ is a graph whose vertices are the primes, such that there is an edge from $p$ to $q$ in $G$ if and only if their base $b$ representations $p=$ $e_{0} b^{0}+e_{1} b^{1}+\ldots+e_{m} b^{m}$ and $q=f_{0} b^{0}+f_{1} b^{1}+\ldots+f_{n} b^{n}\left(0 \leq e_{i}, f_{i}<b\right.$, $e_{m}, f_{n} \neq 0$ ) are such that either $n=m$ and (for some $k$ ) $e_{i}=f_{i}$ for all $i \neq k$ and $\left|e_{k}-f_{k}\right|=1$, or $n=m+1, f_{n}=1$, and $e_{i}=f_{i}$ for $0 \leq i \leq m$.

Some examples are in order. Choosing, for familiarity's sake, $b=10$, we find that there are edges between 2 and 3, and also between 3 and 13, and between 13 and 23, 13 and 113, and 113 and 103. However, although an edge exists from 103 back to 3, this last edge is unidirectional. We cannot go from 3 directly to 103 . 
An exploration of $G_{*}^{10}$ turns out to be less interesting than that of the original prime number maze. The graph seems to have many small disconnected components. Defining the generated subgraph $G_{p}^{b}$ to be the subgraph of $G_{*}^{b}$ containing all vertices $q$ for which a path exists from $p$ to $q$, one finds that $G_{2}^{10}=\{2,3,13,23,103,113,1103\}, G_{5}^{10}=\{5\}, G_{7}^{10}=\{7,17\}$, $G_{11}^{10}=\{11\}$, and so on. The situation seems to improve only slightly as $p$ increases. The largest $G_{p}$ for any $p$ less than 2 million is $G_{262331}$, which has 34 elements, the smallest and largest of which are 262231 and 1111100262101.

A heuristic argument from [6] suggests one reason why this should be the case. Given a large prime $N$, there are of order $2((b-1) / b) \log _{b} N$ numbers which differ from $N$ according to the rules of the maze. A fraction of order $1 / \ln N$ of these should be prime, on average. It follows that there are of order $2((b-1) / b) \ln b$ edges leading out from $N$, on average. This will be less than 1 for all bases $b>4$, hence we may make the following conjecture.

2.1. Conjecture. For no prime $p$ or base $b>2$ is the subgraph $G_{p}^{b}$ of the base $b$ maze infinite.

Reason. For $b>4$, the above heuristic argument shows that the average valence of a vertex should be less than one, making the connected components finite. For $b=4$ and $b=3$, the argument gives average valences greater than 1 , but these expected average valences are sufficiently reduced by the following results to make the conjecture reasonable.

An exploration of the base $b$ mazes for various $b$ reveals that the heuristics are not sufficient to explain the sizes of the components discovered. To shed more light on this matter, we must turn to the next theorem.

First, define the digit sum of $p \in G_{*}^{b}$ to be $\delta_{1}^{b}(p)=e_{0}+e_{1}+\ldots+e_{m}$, where $p=e_{0} b^{0}+e_{1} b^{1}+\ldots+e_{m} b^{m}$ is the base $b$ representation of $p$.

Note that if there is an edge of $G_{*}^{b}$ between $p$ and $q$, then $\delta_{1}^{b}(p)=\delta_{1}^{b}(q) \pm 1$. Note also that if $r$ is any divisor of $b-1$, then $r \mid p$ if and only if $r \mid \delta_{1}^{b}(p)$. This leads immediately to the following:

2.2. THEOREM. If $b>2$, the graph $G_{*}^{b}$ has infinitely many disjoint components.

Proof. Let $P$ be the set of primes dividing $b-1$, and let $N=\left\{\delta_{1}^{b}(p)\right.$ : $p \in P\}$. A path in $G_{*}^{b}-P$ maps to a path in the graph $Z$ whose vertices are the positive integers, and for which there is an edge between $n$ and $m$ if $|n-m|=1$ and $\operatorname{GCD}(n, b-1)=1$ or $n \in N$, and $\operatorname{GCD}(m, b-1)=1$ or $m \in N$. Since (if $b>2$ ) the graph $Z$ has infinitely many disjoint components, so does $G_{*}^{b}$.

In fact, for odd $b$, the graph $Z$ used in the above proof consists mostly of isolated vertices, which means the same is true of $G_{*}^{b}$. This stands to reason, since if $p$ and $q$ satisfy the conditions for them to share an edge, 
and if $b$ is odd, then one of $p$ or $q$ must be even. In this case, the most interesting generated subgraphs would contain at most four vertices: $G_{p}^{b}=$ $\{p, 2,3, b+2\}$, if $b+2$ is prime, and $p=b^{n}+2$ (for some $n$ ) is also prime.

For $b=4$ (and $b=10$ ), the digit sum is forbidden (in general) to be a multiple of 3 . This is also quite restrictive, since it forces us to alternately add 1 and subtract 1 from digits of the numbers we meet as we walk through the maze.

For $b=2$, the digit sum tells us nothing at all about the primality of the number. There appear to be primes with every possible digit sum (except 1).

3. Alternating digit sums. Define the alternating digit sum $\delta_{-1}^{b}(p)$ of $p$ by $\delta_{-1}^{b}(p)=e_{0}-e_{1}+e_{2}-\ldots+(-1)^{m} e_{m}$, where $p=e_{0} b^{0}+e_{1} b^{1}+\ldots+e_{m} b^{m}$ is the base $b$ representation of $b$.

As with the digit sums, we can use alternating digit sums to prove a result about the structure of $G_{*}^{b}$. In this case, we use the fact that for any factor $r$ of $b+1, r$ divides $p$ if and only if $r$ divides $\delta_{-1}^{b}(p)$. This yields a theorem exactly like Theorem 2.2, except that it also applies when $b=2$.

3.1. TheOREM. If $b>1$, the graph $G_{*}^{b}$ has infinitely many disjoint components.

Proof. The proof is almost identical to that of Theorem 2.2, but uses $\delta_{-1}^{b}$ and $b+1$ instead of $\delta_{1}^{b}$ and $b-1$.

This result, along with Theorem 2.2 and the heuristics which precede it, suggest that the mazes with the largest components will be those base $b$ mazes for which $b$ is not large, and neither of $b \pm 1$ have small prime factors. Indeed, the base 12 maze has many large components. The smallest $p$ for which $\left|G_{p}^{12}\right|>100$ is only $p=6907_{10}=3 \mathrm{BB} 7_{12}$. The elements of $G_{6907}^{12}$, numbering 122 in all, range from $991_{10}=6 \mathrm{~A} 7_{12}$ to $5593001719_{10}=$ $1101102987_{12} . G_{58453}^{12}$ has 154 elements.

For the original prime number maze, with $b=2$, Theorem 3.1 allows us to make the following definitions. Let the $k$-partition $\Gamma_{k}$ of $G_{1}=G_{*}^{2}$ be the set of all primes $p$ such that $\delta_{-1}^{2}(p)$ equals $3 k+1$ or $3 k+2$.

3.2. Theorem. Let $p \in \Gamma_{i}$ and $q \in \Gamma_{j}$. If there is a path in $G_{1}$ from $p$ to $q$, then either $i=j$, or $\{i, j\}=\{-1,0\}$ and the path contains the number 3 .

Proof. When $b=2$, the alternating digit sum $\delta_{-1}^{2}(p)$ is a multiple of 3 if and only if $p$ is a multiple of 3 . Therefore, if $p^{\prime} \in \Gamma_{i}$, and there is an edge in $G_{1}$ between $p^{\prime}$ and $q^{\prime}$, then either $q^{\prime}=3$, or $q^{\prime} \in \Gamma_{i}$ also. Therefore, it is impossible for a path from $p \in \Gamma_{i}$ to leave $\Gamma_{i}$ except via 3 . The fact that 3 is only reachable from $\Gamma_{-1}$ and $\Gamma_{0}$ completes the proof.

Note that the $\alpha-, \beta-, \gamma$ - and $\delta$-partitions as defined in [6] more or less correspond to $\Gamma_{0}, \Gamma_{-1}, \Gamma_{1}$ and $\Gamma_{-2}$ respectively. However, [6] places 2 in the 
$\alpha$-partition, but the fact that $\delta_{-1}^{2}(2)=-1=3(-1)+2$ places it in $\Gamma_{-1}$. The prime 2 is anomalous in this respect, in that the only edge from or to 2 passes directly through 3 , and the only edges out of 3 lead to 2 or into $\Gamma_{0}$, so that $G_{2}-\{2,3\} \subset \Gamma_{0}$.

Note also that the partitions $\Gamma_{k}$ cover all primes except 3, whereas the partitions of [6] exclude isolated primes, and other finite subgraphs of $G_{1}$ (if any exist).

Paulsen was unable to discover to which of his partitions ( $\beta$ - or $\gamma$-) the prime 6379 belongs. Using the fact that $6379=1100011101011_{2}$, we can deduce that if it is in either, it must be in the $\beta$-partition, since $\delta_{-1}^{2}(6379)$ $=-2$.

The partitions $\Gamma_{k}$ are strongly linked to the concept of parity.

3.3. TheOREM. A prime $p>3$ has correct parity if and only if it is in $\Gamma_{k}$ for some even $k$.

Proof. Note that $\delta_{1}^{2}(p)$ and $\delta_{-1}^{2}(p)$ are either both even or both odd. If $p$ has correct parity, then if $\delta_{1}^{2}(p)$ is even, so is $\delta_{-1}^{2}(p)=3 k+2$, and if $\delta_{1}^{2}(p)$ is odd, so is $3 k+1$. Either way, $k$ must be even. Conversely, if $p$ has incorrect parity, $k$ must be odd.

Table 2. The partitions $\Gamma_{k}$

\begin{tabular}{|l|c|c|}
\hline$\Gamma_{k}$ & Minimum prime & Starting point \\
\hline$\Gamma_{0}$ & 5 & 5 \\
$\Gamma_{-1}$ & 2 & 547 \\
$\Gamma_{1}$ & 277 & 4,957 \\
$\Gamma_{-2}$ & 683 & 35,759 \\
$\Gamma_{2}$ & 17,749 & 82,261 \\
$\Gamma_{-3}$ & 43,691 & $2,271,403$ \\
$\Gamma_{3}$ & $1,398,037$ & $55,923,157$ \\
$\Gamma_{-4}$ & $2,796,203$ & $145,402,811$ \\
$\Gamma_{4}$ & $72,701,269$ & $3,310,702,933$ \\
$\Gamma_{-5}$ & $715,827,881$ & $28,624,724,651$ \\
$\vdots$ & $\vdots$ & $\vdots$ \\
\hline
\end{tabular}

Table 2 gives the minimum prime, and the "starting point" for various $\Gamma_{k}$. The "starting point" is loosely defined as the smallest prime $r$ in the partition for which $G_{r}^{\prime}$ "appears" to be infinite. The reason for using $G_{r}^{\prime}$ instead of $G_{r}$ is to exclude starting points such as 11: $G_{11}$ appears to be infinite, but only contains two elements from the partition containing 11 . The starting points were identified as the smallest element $p$ of each $\Gamma_{k}$ for 
which $\left|G_{p}^{\prime}\right| \geq 10000$. By way of comparison, the largest $G_{p}$ proven finite during this search had less than 100 elements.

4. Generalizations. The digit sum and the alternating digit sum can be generalized as follows.

Let $\omega$ be the root $\cos (2 \pi / n)+i \sin (2 \pi / n)$ of the $n$th cyclotomic polynomial $\Phi_{n}(x)$, and let $\mathbb{Z}[\omega]$ be the ring of algebraic integers of the extension field $\mathbb{Q}(\omega)$ of $\mathbb{Q}$. We shall concentrate here primarily on the case where $\mathbb{Z}[\omega]$ is a unique factorization domain. Define the $\omega$-digit sum $p \delta=\delta_{\omega}^{b}(p)$ to be

$$
\sum_{k=0}^{m} e_{k} \omega^{k},
$$

where $p=e_{0} b^{0}+e_{1} b^{1}+\ldots+e_{m} b^{m}$ is the base $b$ representation of $p$ ( $p$ need not be a prime number here).

It is desirable to find a map $\psi: \mathbb{Z}[\omega] \rightarrow \mathbb{Z}_{r}=\mathbb{Z} / r \mathbb{Z}$ for some $r$, such that $p \delta \psi=0+r \mathbb{Z}$ if and only if $r \mid p$. Then a walk through the prime maze becomes a walk through $\mathbb{Z}[\omega]$, with each step of length 1 , and ker $\psi \subseteq \mathbb{Z}[\omega]$ becomes a set of forbidden points in the latter walk, just as the multiples of primes dividing of $b-1$ or $b+1$ were forbidden points when $\omega$ was 1 or -1 .

To achieve this goal, let $k=\phi(n)$ be the degree of $\Phi_{n}(x)$, and let $r$ be a divisor of $\Phi_{n}(b)$. Any element of $\mathbb{Z}[\omega]$ may be uniquely written as $\chi=e_{0}+e_{1} \omega+\ldots+e_{k-1} \omega^{k-1}$. Let $\psi$ map $\chi$ to $e_{0}+e_{1} b+\ldots+e_{k-1} b^{k-1}+r \mathbb{Z}$.

4.1. Theorem. The map $\psi$ defined above is a well defined ring homomorphism. Furthermore, for $p \in \mathbb{Z}, r \mid p$ if and only if $p \delta \in \operatorname{ker} \psi$.

Proof. Certainly it is well defined. It is easy to show that it preserves addition. Now, if $\chi_{1}=e_{0}+e_{1} \omega+\ldots+e_{k-1} \omega^{k-1}=e(\omega)$ and $\chi_{2}=f_{0}+f_{1} \omega+$ $\ldots+f_{k-1} \omega^{k-1}=f(\omega)$ are such that $\chi_{1} \chi_{2}=g_{0}+g_{1} \omega+\ldots+g_{k-1} \omega^{k-1}=$ $g(\omega)$ where $e(x), f(x), g(x) \in \mathbb{Z}[x]$, it can only be because the cyclotomic polynomial $\Phi_{n}(x)$ divides $g(x)-(e f)(x)$ in the polynomial ring $\mathbb{Z}[x]$. It follows then that $\Phi_{n}(b)$ divides $g(b)-(e f)(b)$, whence $\left(\chi_{1} \psi\right)\left(\chi_{2} \psi\right)=\left(\chi_{1} \chi_{2}\right) \psi$ as required.

Now, if $p=e_{0}+e_{1} b+\ldots+e_{m} b^{m}$, then $p \delta \psi=e_{0}+e_{1} b+\ldots+e_{m} b^{m}+r \mathbb{Z}=$ $p+r \mathbb{Z}$, so $p \delta \psi=0+\mathbb{Z}$ if and only if $r$ divides $p$, as required.

As an example, let $n=4$, so that $\omega=i=\sqrt{-1}$, and $\mathbb{Z}[\omega]$ becomes the Gaussian integers $\mathbb{Z}[i]$. The cyclotomic polynomial $\Phi_{n}(x)$ in this case is $x^{2}+1$, so if $b=2$, we must use $r=5$. Let $c+i d \in \mathbb{Z}[i]$. Then $(c+i d) \psi=c+2 d$, which is a multiple of 5 if and only if $c+2 d$ is a multiple of the Gaussian prime $1+2 i$. A walk through $G_{1}$ must avoid numbers $p>5$ such that $1+2 i$ divides $\delta_{i}^{2}(p)$. 
At first glimpse, this is not a very useful fact. Consideration of $\delta_{1}^{b}$ and $\delta_{-1}^{b}$ yielded powerful results because the corresponding cyclotomic polynomials $\Phi_{1}(x)$ and $\Phi_{2}(x)$ each have degree 1 , so that the walk through $\mathbb{Z}[\omega]$ is through $\mathbb{Z}$ itself. Such a walk is already sufficiently constrained that the extra constraint(s) (that the walk must not touch ker $\psi$ ) splits the maze into infinitely many partitions. Unfortunately, a walk in $\mathbb{Z}[i]$ has so much freedom that the restriction on $\delta_{i}^{2}(p)$ (that it should not be a multiple of $1+2 i)$ is not enough to help very much.

On the other hand, for any prime $r$ not dividing $b, r$ divides $b^{r-1}-1$, so that there exists some $n$ dividing $r-1$ for which $r \mid \Phi_{n}(b)$. It follows that any condition of the form " $r$ must not divide $p$ " can be expressed in the form " $\delta_{\omega}^{b}(p)$ must not be in ker $\psi$ ", for some $\omega$, no matter what the base $b$ under consideration. Therefore, the above analysis (almost) completely captures the structure of the prime number maze (except that $\delta_{\omega}^{b}(p)$ is permitted to touch ker $\psi$ for the one exception $p=r$ ).

Furthermore, for at least some bases $b$, these results do produce some "useful" partitioning theorems. A method for finding them is illustrated in Section 5, after the following lemmas and theorem.

4.2. LEMmA. Let $n$ be such that $\mathbb{Z}[\omega]$ is a unique factorization domain, let $r$ be a prime integer dividing $\Phi_{n}(b)$, and define the maps $\delta=\delta_{\omega}^{b}$ and $\psi$ as before. Then $r$ factorizes over $\mathbb{Z}[\omega]$, and $\chi \psi=0+r \mathbb{Z}$ for precisely one of its irreducible factors (up to associates).

Proof. Now, if $\chi_{1} \psi=\chi_{2} \psi=0+r \mathbb{Z}$, then $\operatorname{GCD}\left(\chi_{1}, \chi_{2}\right) \psi=0+r \mathbb{Z}$ also, which leads to a contradiction if $\operatorname{GCD}\left(\chi_{1}, \chi_{2}\right)$ is a unit of $\mathbb{Z}[\omega]$. Since $r \psi=(b-\omega) \psi=0+r \mathbb{Z}$, and $b-\omega$ is not a multiple of $r$, it follows that $r$ is not irreducible in $\mathbb{Z}[\omega]$. Let $r=\chi_{1} \chi_{2} \ldots \chi_{l}$ be a factorization of $r$ in $\mathbb{Z}[\omega]$ into irreducibles. It follows that $\chi_{i} \psi=0+r \mathbb{Z}$ for exactly one of the $\chi_{i}$. It must be the case for at least one, since $\left(\chi_{1} \psi\right)\left(\chi_{2} \psi\right) \ldots\left(\chi_{l} \psi\right)=0+r \mathbb{Z}$, and $\mathbb{Z}_{r}$ is a field $\left(r\right.$ being prime in $\mathbb{Z}$ ). If $\chi_{i} \psi=\chi_{j} \psi=0+r \mathbb{Z}$, where $\chi_{i}$ and $\chi_{j}$ are not associates, then the fact that $\operatorname{GCD}\left(\chi_{i}, \chi_{j}\right)$ is a unit leads to a contradiction.

4.3. Lemma. Let $n$ be as before, let $r$ be a prime integer dividing $\Phi_{n}(b)$, and let $\chi$ be an irreducible factor of $r$ in $\mathbb{Z}[\omega]$. Let $G$ be the Galois group of $\mathbb{Q}(\omega)$, and let $X=\chi^{G}$ be the orbit of $\chi$ under the action of the Galois group. Then $r=\prod_{\zeta \in X} \zeta$.

Proof. First of all, let $\chi$ divide $r$. Then, for any element $\alpha$ of the Galois group, $\chi \alpha$ divides $r \alpha=r$. Hence every element of $X$ divides $r$, so $\prod_{\zeta \in X} \zeta$ divides $r$. If $r$ has some additional nontrivial factor $v$, then letting $Y=v^{G}$, we find that $\left(\prod_{\zeta \in X} \zeta\right)\left(\prod_{v \in Y} v\right)$ divides $r$, contradicting the choice of $r$ as a prime integer. 
4.4. THEOREM. Let $n$ and $r$ be as before, and let $\psi_{b}$ be the map $\psi$ defined earlier, with $\omega \psi_{b}=b+r \mathbb{Z}$. Then for each irreducible factor $\chi$ of $r$ there exists $b^{\prime}$ such that $\chi \psi_{b^{\prime}}=0+r \mathbb{Z}$.

Proof. From Lemma 4.2, there exists $\chi_{1}$ such that $\chi_{1} \psi_{b}=0+r \mathbb{Z}$, and from Lemma 4.3, there exists an element $\alpha$ of the Galois group of $\mathbb{Q}(\omega)$ such that $\chi \alpha=\chi_{1}$. Now $\alpha$ sends $\omega$ to a primitive $n$th root of unity, that is, $\omega \alpha=\omega^{i}$ for some $i$ with $\operatorname{GCD}(i, n)=1$ (see Chapter 48 of [3], in particular Theorem 48.1). It follows that $\alpha \psi_{b}=\psi_{b^{i}}$, and hence, letting $b^{\prime} \equiv b^{i} \bmod r$, that $\chi \psi_{b^{\prime}}=\chi \psi_{\left(b^{i}\right)}=\chi \alpha \psi_{b}=\chi_{1} \psi_{b}=0+r \mathbb{Z}$, as required.

5. Some final partition theorems. The main motivation for the results of the previous section is to prove that the technique illustrated here will always work (at least when $\mathbb{Z}[\omega]$ is a unique factorization domain).

Let $n=4$, that is, let $\omega=\sqrt{-1}$, so $\mathbb{Z}[\omega]$ is the set $\mathbb{Z}[i]$ of Gaussian integers. Figure 1 shows in black those Gaussian integers which are divisible by either $2-i, 3-2 i, 4-i, 5-2 i$ or $4-5 i$.

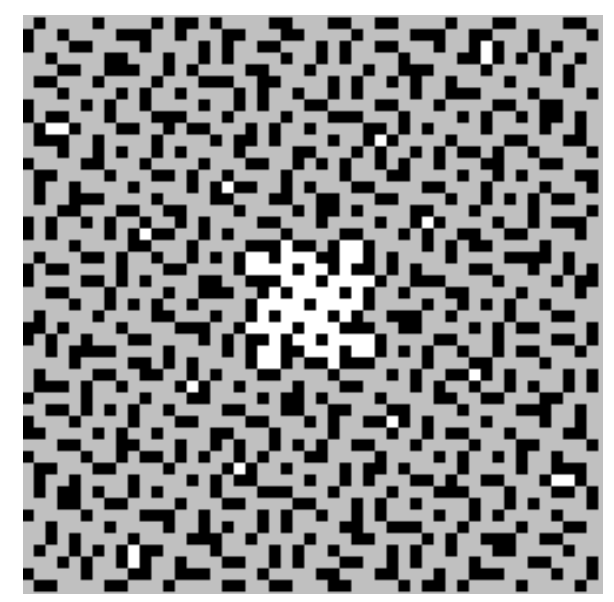

Fig. 1. The Gaussian integers

It will be noted that there is a pinwheel-shaped region centered at $0+0 i$ which is separated from the rest of the plane by a "wall" of multiples of these primes. If we can find a base $b$ for which $5|(2-b), 13|(3-2 b), 17 \mid(4-b)$, $29 \mid(5-2 b)$ and $41 \mid(4-5 b)$, then we will have another proof of the following theorem.

5.1. TheOREM. For such a $b$, the base $b$ maze has infinitely many disjoint partitions. 
Proof. Infinitely many, because even though the boundary of the pinwheel centered at $0+0 i$ contains 5 , the pinwheel pattern is repeated around every multiple in $\mathbb{Z}[i]$ of $(2-i)(3-2 i)(4-i)(5-2 i)(4-5 i)=-966-617 i$.

The partitions derived from the pinwheel will be quite distinct from any partitions derived using Theorems 2.2 and 3.1 (digit sums or alternating digit sums).

Let us proceed to find such a base $b$. If $b$ is as required, then $b$ is a solution to the congruences

$$
\begin{aligned}
& 2-b \equiv 0 \bmod 5, \quad \text { that is, } \quad b \equiv 2 \bmod 5, \\
& 3-2 b \equiv 0 \bmod 13, \quad \text { that is, } b \equiv 8 \bmod 13 \text {, } \\
& 4-b \equiv 0 \bmod 17, \quad \text { that is, } b \equiv 4 \bmod 17 \text {, } \\
& 5-2 b \equiv 0 \bmod 29, \quad \text { that is, } b \equiv 17 \bmod 29 \text {, } \\
& 4-5 b \equiv 0 \bmod 41, \quad \text { that is, } b \equiv 9 \bmod 41 \text {. }
\end{aligned}
$$

It follows that $b \equiv 1103032 \bmod 1313845$.

In fact, for some such bases $b$ (in particular $b=1103032+1313845 k$, where $k=26,86, \ldots)$, both $b+1$ and $b-1$ are prime, so that this analysis yields a partition theorem which is stronger (in some sense) than either of Theorems 2.2 or 3.1 .

As a bonus, this figure also shows some points (namely $6+14 i, 10+7 i$ and their associates) which are completely surrounded by black. These yield the equivalent of Brier numbers in these bases. Specifically, if $\delta_{i}^{b}(k)=6+14 i$ or $10+7 i$, then $k \pm b^{m}$ is composite for any $m$, and from whence it may be shown that $k \cdot b^{n} \pm 1$ is also composite for any $n$. The smallest such example is $k=7721234$ for $b=1103032$, so that $7721234 \cdot 1103032^{n} \pm 1$ is always composite, being divisible by one of $5,13,17,29$ or 41 . Note that one should not expect that all Brier numbers for all bases will be obtainable in this way.

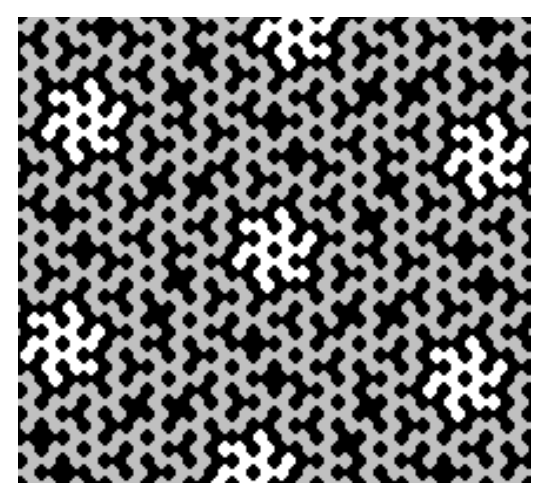

Fig. 2. The Eisenstein integers 


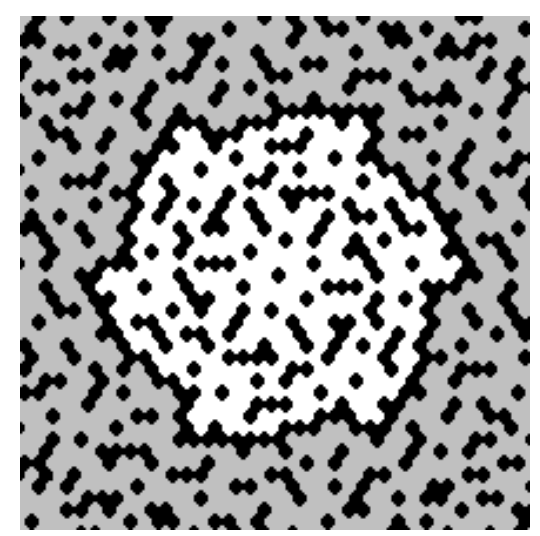

Fig. 3. The Eisenstein integers again

Alternatively, by considering the complex conjugate diagram to Figure 1, one can deduce partition theorems for bases of the form $b=210813+$ $1313845 k$. There are twin primes $b \pm 1$ for $b$ of this form when $k=15,39$, $183, \ldots$, and one may obtain the generalized Brier number 1264892 for $b=$ 210813.

Figures 2 and 3 show elements of $\mathbb{Z}[\omega]$, where $\omega=\frac{1}{2}(1+i \sqrt{3})$ is a primitive sixth root of 1 (the so-called Eisenstein integers). In Figure 2, the black dots are those elements which are multiples of either $1+\omega, 1+2 \omega$ or $3+\omega$. Again, there are repeated pinwheels isolated from the rest of $\mathbb{Z}[\omega]$ by walls of multiples. This provides a partition theorem for bases $b$ satisfying $3|(1+b), 7|(1+2 b)$ and $13 \mid(3+b)$, that is, $b$ is of the form $b=101+273 k$. Alternatively, since $\mathbb{Z}[\omega]=\mathbb{Z}\left[\omega^{2}\right]$ and $\omega^{2}$ is a primitive cube root of 1 , we can write $1+\omega=2+\omega^{2}, 1+2 \omega=3+2 \omega^{2}$ and $3+\omega=4+\omega^{2}$, and then find a partition theorem for bases $b$ satisfying $3|(2+b), 7|(3+2 b)$ and $13 \mid(4+b)$, that is, $b=100+273 k$.

In fact, $\Phi_{3}(100)=10101=3 \cdot 7 \cdot 13 \cdot 37$, so if we let $b=100$ and $r=37$ and define the map $\psi$ as before, there exists also a factor $\alpha$ of 37 in $\mathbb{Z}\left[\omega^{2}\right]$ for which $\alpha \psi=0+37 \mathbb{Z}$. The factor in question may be taken to be $7+4 \omega^{2}$. Adding all multiples of $7+4 \omega^{2}$ to Figure 2 would reveal some isolated points in the prime number maze, leading also to generalized Brier numbers for base 100. The smallest generalized Brier number obtainable in this way is 926 . It should be noted that this is by no means the smallest generalized Brier number for base 100, which is 10 (or 43, if one insists that $k \cdot b^{n} \pm 1$ be composite for $n=0$ also).

Figure 3 shows a larger pinwheel bounded by multiples of $2+\omega, 1+3 \omega$, $1+5 \omega, 4+3 \omega, 6+\omega, 4+5 \omega, 6+7 \omega, 9+5 \omega, 1+12 \omega$ and $11+3 \omega$. It is included to show that multiples of $1+\omega$ are not strictly necessary. The solution of the resulting system(s) of congruences is left as an exercise. 
The author readily admits that few human beings are likely to explore the base 1103032 or 210813 prime number maze as a form of recreation or for any pragmatic purpose (although the fortuitous base 100 theorem may be of some interest). The same methods could be applied to find partition theorems based on any cyclotomic extension $\mathbb{Z}[\omega]$. For higher-degree extensions, more irreducibles will be needed, in general making the obtained base even higher. It seems unlikely to this author that for the base 2 maze, any useful partition theorem beyond Theorem 3.1 can be obtained.

6. Concluding remarks. There are other questions that may be worth investigating. The base $b$ maze was defined so that at every step, one digit was changed by \pm 1 . One could also define the mazes by allowing larger changes. For example, at each step we could allow a digit to change by $\pm t$, for any $0<t \leq k \leq b-1$ ( $k$ fixed). Or, we could define a maze on the Gaussian primes, allowing at each step a change in one of the digits of either the real or the imaginary part.

Some other somewhat similar explorations were suggested by the referee. For example, if we allow digits to be added and removed, but not changed, we obtain what are called "truncatable" primes. A prime is left-truncatable if one can delete any number of its lead digits, and still obtain a prime. Similarly defined are right-truncatable primes. A deletable prime is one where for any $k$ less than the number of its digits, we can remove some set of $k$ digits and still have a prime. There are only finitely many left- and righttruncatable primes, but it is conjectured that the number of deletable primes is infinite. See [1] or [2] for information about these (and other) numbers.

The referee also pointed out some references to the "Gaussian Moat Problem". If one defines a walk on the primes such that at each step from $p_{1}$ to $p_{2}$ has $\left|p_{1}-p_{2}\right|$ less than some fixed length, the walk is bounded, since arbitrarily large gaps exist in the primes. The equivalent problem is unsolved on the Gaussian primes, although some work has been done on it (see [4], [5] and [7]). However, there is apparently no work published on the "Eisenstein Moat Problem". It seems that whatever techniques eventually solve the former, they will likely apply just as well to the latter.

\section{References}

[1] C. Caldwell, Truncatable primes, J. Recreational Math. 19 (1987), 30-33.

[2] L. E. Card, Patterns in primes, ibid. 1 (1968), 93-99.

[3] J. B. Fraleigh, A First Course in Abstract Algebra, Addison-Wesley, 1982.

[4] E. Gethner and H. M. Stark, Periodic Gaussian moats, Experiment. Math. 6 (1997), 289-292.

[5] E. Gethner, S. Wagon and B. Wick, A stroll through the Gaussian primes, Amer. Math. Monthly 105 (1998), 327-337. 
[6] W. Paulsen, The prime number maze, Fibonacci Quart., to appear.

[7] I. Vardi, Prime percolation, Experiment. Math. 7 (1998), 275-289.

Sepang Institute of Technology

Level 5, Klang Parade

2112 Jalan Meru

Klang, 41050, Selangor, Malaysia

E-mail: Michael.Hartley@sit.edu.my

Received on 12.7.2001

and in revised form on 23.1.2002 